\title{
La gestión del conocimiento como estrategia en el trabajo del documentalista
}

\author{
Anne-Vinciane Doucet \\ María José Sánchez García \\ Universidad de Granada (España)
}

We can know more

than we can tell

Polyni

\section{Resumen}

El cambio provocado por el desarrollo de las nuevas tecnologías de la información y comunicación (NTIC) ha dado lugar a una nueva sociedad denominada de la información, que a su vez, enmarcada dentro de la gestión de los nuevos recursos, se denomina del conocimiento. Es dentro de este entorno donde se plantean los grandes problemas que afectan a dicho movimiento, ya que cada vez se genera más información, con mayor rapidez y sin ningún tipo de control. El medio con mayor difusión, Internet, da la oportunidad de que cualquier usuario pueda tener acceso desde cualquier lugar, provocando un nuevo entorno de trabajo en el documentalista que se convierte en una herramienta clave, ya que es el encargado de gestionar todo el contenido mediático y proporcionar a la información un valor añadido que permite al receptor establecer el proceso de feedback rápidamente. Esta es la razón por la que el documentalista sigue siendo el intermediario entre la información y el usuario, pero además es el encargado de enseñar a gestionar dicha información. En este entorno, es vital desarrollar estructuras con el fin de organizar y representar el conocimiento de forma adecuada, tanto para su principal receptor (usuario/cliente) como para el profesional que se encarga de ello. Para ambos, la gestión del conocimiento se convierte en un factor clave en el desarrollo de la actividad. El presente trabajo pretende estudiar el proceso de gestión y organización del conocimiento como estrategia en el desempeño de las tareas documentales del profesional de la información, tanto en su faceta estratégica y de toma de decisiones como en el desarrollo de procesos y productos.

Palabras clave: Gestión del conocimiento. Organización del conocimiento. Profesional de la información. 


\section{Abstract}

The change brought about by new information and communication technologies (NICT) has given rise to a new society, the so-called information society, which, in its turn, within the frame of the management of new resources, is called the knowledge society. It is in this context that serious problems arise affecting this movement, since more and more information is generated ever more quickly and without any kind of control. The most widespread medium, Internet, provides access to any user anywhere. This has created a new work context for the library and information scientist who becomes a key instrument, as s/he is in charge of managing all the mediatic content and enhancing information to enable the user to give rapid feedback. For this reason the library scientist is still the intermediary between information and the user. What is more, s/he is also responsible for the teaching of information management. In this environment, it is vital to develop structures for the organisation and representation of knowledge in a suitable way, both for the receptor (the user/client) and for the professional who has to deal with it. For both, how knowledge is organised is of paramount importance for the success of his/her activity. The aim of the present study is to examine the process of managing and organising knowledge as a strategy in the information manager's documentary tasks, in his/her strategic and decision-making roles, as well as in his/her development of processes and products.

Keywords: Knowledge management. Knowledge organization. Information manager.

\section{Introducción}

Al siglo XXI se le conoce como el de la globalización, de la información y del conocimiento; se habla de la economía mundial (Drucker, 1996), que consta de los movimientos de dinero e información, y por otro lado de comercio e inversiones. La economía estructurada de forma organizada pasa a ser estructurada en torno a la información. Con Internet cualquiera puede acceder desde cualquier lugar a cualquier tipo de información en cualquier idioma. En las organizaciones la información sustituye a la autoridad, y se vuelve factor clave para el desarrollo competitivo de las mismas.

La sociedad del conocimiento se caracteriza por una abundancia de información que es necesario organizar, y las nuevas tecnologías de la información y la comunicación son un ejemplo. Son muchos los autores que piensan que la gestión del conocimiento se ha desarrollado a partir del auge de las nuevas tecnologías, pero se trata de una idea equivocada, ya que todas las organizaciones han manejado siempre gran cantidad de conocimiento. El problema se plantea en los métodos para gestionarlo y el valor competitivo que se le ha atribuido en los últimos años. Dicho desarrollo lleva consigo un gran aumento del conocimiento, que hace necesaria la existencia de unos profesionales encargados de gestionar y enfrentarse diariamente 
al gran flujo de información y conocimiento que inunda la sociedad actual. Para ello se han tenido que crear herramientas que permitan una gestión óptima y lleven a cabo estrategias de trabajo competitivo.

El que trabajo que presentamos a continuación se centra en la investigación y propuesta de las nuevas herramientas de trabajo de las que dispone el documentalista o profesional de la información para realizar una correcta gestión.

\section{La gestión del conocimiento}

La gestión del conocimiento se enmarca dentro de un proceso evolutivo; se puede considerar como un escalón óptimo al que el ser humano debe llegar para seguir evolucionando. Partiendo de la trilogía base dato-información-conocimiento, se entabla una cadena evolutiva donde los datos obtenidos de los documentos (imágenes, letras, etcétera), al procesarlos y presentarlos de una forma estructurada (esquemas, bases de datos, etcétera), se convierten en información. Para ascender desde este nivel hasta el del conocimiento es necesario pasar por una etapa intelectual única donde la información se comprende y se añade al conocimiento de la persona o institución que la interpreta. Así, el conocimiento considerado como habilidad y capacidad solo puede ser transferido por medio de una interacción única interpersonal o interinstitucional (Al-Hawamdeh, 2002) (fig. 1).

Desde el punto organizacional, la corriente que lleva a buen puerto el barco del conocimiento es el capital humano; en él recae la responsabilidad de que la información almacenada se comprenda en todos los sentidos. Edvinsson y Malone

\section{Conocimiento}

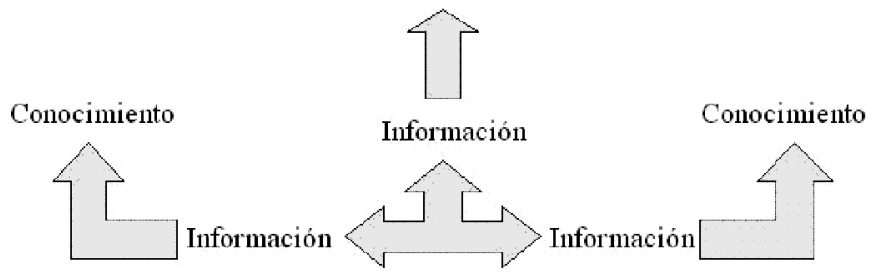

Conocimiento

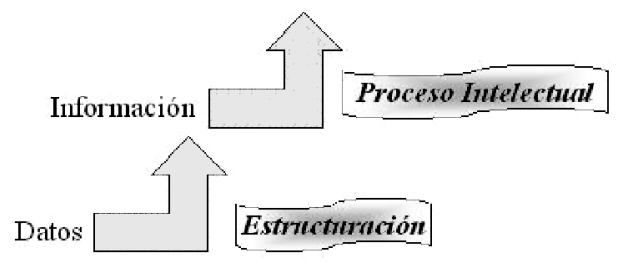

Figura 1. Bifurcación del proceso del conocimiento.

Scire. $14: 1$ (en.-jun. 2008) 75-86. ISSN 1135-3716. 
lo definen como una información complementaria (García Jiménez, 2002). En todas las organizaciones, el capital intelectual debe reunir las competencias, las capacidades y el saber de sus miembros, las estructuras, los procesos y las rutinas creadas por estos durante su trabajo y en su entorno (Esteban y Navarro, 2003). De esta forma, el capital intelectual se puede subdividir en capital humano, que comprendería los conocimientos, habilidades, experiencias, etcétera, del personal interno de la organización; capital estructural, considerado como aquel que produce los equipos, programas, bases de datos y todo lo que forma parte de la capacidad organizacional de una empresa; y capital cliente, fruto del desarrollo de las relaciones con los clientes, clave para el éxito (Bustelo y Amarilla, 2001).

La gestión del conocimiento es el proceso que pretende proporcionar herramientas útiles para que la información almacenada se convierta en conocimiento con el fin de aumentar la competitividad en la organización y ayude a sus miembros a tomar decisiones y resolver problemas estratégicos.

El proceso de gestión del conocimiento tiene cuatro pasos fundamentales: generación y captura de la información, organización y almacenamiento de esta información de una forma estructurada y accesible para los miembros de la organización, difusión y transferencia del conocimiento, y aplicación y utilización de este (García-Caso, 2002).

\subsection{Gestión de la información y gestión del conocimiento}

La información y el conocimiento son conceptos que siempre han estado unidos, pero no por ello se deben confundir como sinónimos. Está claro que para llegar a obtener conocimiento en cualquier situación es necesario previamente obtener información de la misma, aunque esto no significa que al tener más información se logre más conocimiento. Condicionar este hecho a la obtención de conocimiento es un error que se comete a menudo en nuestro entorno. Por ello, es necesario dejar claro que, en los sistemas de información, a través del almacenamiento y estructuración de la información se encuentra la base de lo que posteriormente será el sistema de conocimiento, siendo este un proceso individual e intransferible.

\subsection{Herramientas que permiten llevar a cabo un sistema de gestión del conocimiento}

Un sistema de gestión del conocimiento debe utilizar herramientas que hagan posible la correcta gestión de la información, la estructuración de la documentación generada, la distribución de los flujos, etcétera. Para esto siempre se ha contado con sistemas documentales como bases de datos, servicios de alerta o difusión selectiva de la información, entre otros. Pero ha sido el gran avance experimentado en el sector de las nuevas tecnologías el que ha permitido el desarrollo completo de herramientas muy sofisticadas, tales como la gestión electrónica de documentos, el groupware, el e-learning, etcétera (Balmisse, 2003).

Scire. $14: 1$ (en.-jun. 2008) 75-86. ISSN 1135-3716. 


\subsubsection{El groupware}

El groupware, también llamado trabajo en grupo, es un software que permite comunicar, colaborar y coordinar el trabajo entre profesionales, independientemente de su distribución geográfica. Los elementos fundamentales para constituir un groupware son una base de datos documental, que permite a los usuarios reunir, compartir y administrar una gran variedad de documentos; un sistema integrado de mensajería para enviar y recibir correo electrónico y para mover documentos a través de un flujo de trabajo; y un ambiente de desarrollo para crear rápidamente aplicaciones estratégicas basadas en documentos e información estructurada. Estos elementos se apoyan en tres pilares básicos: la comunicación - los miembros del grupo intercambian información-, la colaboración —-mediante la comunicación los miembros del grupo pueden realizar aportaciones, intercambiar opiniones, generar documentos, etcétera-y la coordinación —entre los distintos miembros-

\subsubsection{El workflow}

El workflow o flujo de trabajo consiste en aplicaciones tecnológicas que permiten automatizar los procesos de trabajo desarrollados en una organización. Su implantación es adecuada en cualquier tipo de entorno, y se usa principalmente para coordinar y gestionar el trabajo de grupos de agentes, encaminar automáticamente los procesos de trabajo entre los agentes, controlar, seguir y administrar los procesos de trabajo, conseguir un servicio más rápido y acelerar los ciclos de desarrollo de productos y procesos (Caro et álii, 2002).

Los sistemas workflow permiten especificar los flujos de trabajo en cuatro niveles: nivel de gestión, nivel formal, nivel de automatización y nivel de demostración.

\subsubsection{El e-learning}

Se trata de un sistema que permite que cada miembro pueda mejorar y ampliar sus conocimientos, y luego compartirlos con los demás. Tres elementos definen el e-learning: comunicación, conocimiento y tecnología. Y los tres factores que lo caracterizan son la gestión de contenido, el servicio y la implantación de una plataforma tecnológica que permita la administración de los distintos recursos. De esta forma, un alumno y un profesor pueden, por medio de sus claves, trabajar en el mismo entorno pero en sesiones distintas, compartiendo información. Es importante tener siempre en cuenta que el e-learning es un proyecto, no un producto; se trata de un proceso, y la tecnología es un apoyo al proyecto.

En general, lo que se pretende es crear una red de conocimiento dinámico que ofrezca una formación continua e individualizada. Estas características, unidas al ahorro de tiempo y la supresión de las distancias, han dado lugar a que sean muchas las empresas que han puesto en marcha proyectos para permitir a sus usuarios (tanto

Scire. $14: 1$ (en.-jun. 2008) 75-86. ISSN 1135-3716. 
los externos como los propios trabajadores) mantenerse actualizados a través del entorno virtual (1).

\subsubsection{Mapas conceptuales}

Se pueden definir como una representación gráfica de un conjunto de conceptos y sus relaciones sobre un dominio específico de conocimiento, construido de tal forma que las interrelaciones entre los conceptos son evidentes (Sánchez y Doucet, 2004).

Los mapas conceptuales (Cañas et álii, 2000) fueron desarrollados por Novak y Gowin (1988), quienes definían los cuatro elementos fundamentales de estos: el profesor, el que aprende, el vitae y el medio. Están basados en la teoría de aprendizaje de David Ausubel, que centra la atención en los conceptos y en el aprendizaje proposicional como base sobre la que construyen los individuos sus significados propios e idiosincrásicos; es decir, se basan en la teoría de la asimilación, según la cual el alumno demuestra lo que sabe y construye su propio conocimiento a través de la experiencia. En definitiva, se puede decir que los mapas conceptuales son un recurso esquemático para representar un conjunto de significados conceptuales y al final proporcionan un resumen de todo lo aprendido.

\section{El profesional de la información}

Trataremos al profesional de la información en general, ya sea documentalista de una empresa, de un colegio/instituto o de un centro de documentación, pues, según la Association des Professionnels de l'Information et de la Documentation (ADBS), existen 29 profesiones tipo de documentalista.

Silva y Cunha (2002) subrayan que "una de las cuestiones centrales de la sociedad de la información es que el objeto del trabajo del hombre pasa a ser la interacción con hombres. El saber y la comunicación pasan a ocupar la mayoría de las actividades humanas". También indican que "los bibliotecarios, profesionales que privilegian la información en su hacer cotidiano, poseen un papel importante a cumplir en la sociedad del conocimiento", lo que significa que los documentalistas se han vuelto "piezas clave" en la sociedad del conocimiento. Vemos una evolución en el trabajo del documentalista. Vamos a centrarnos en sus funciones para ver cómo la gestión del conocimiento puede ser una estrategia en su trabajo.

\subsection{Misión}

Se trata de unas breves líneas que definen el propósito final de la empresa. Trasladándonos al ámbito de la documentación y tomando prestadas las palabras de Jacques Attali, definiremos la misión del profesional de la información no ya como la de poner a disposición la información, sino la de jerarquizarla y darle sentido (Accart, 2000), o sea, añadirle valor. Attali dice además que "producir sentido es sin duda 
el futuro de esta profesión". La información ya no está jerarquizada de forma tradicional (dentro de la empresa, se entiende), sino que se ha vuelto transversal.

Con esta definición nos damos cuenta de que el papel del profesional de la información ha evolucionado: ya no se trata solo de poner a disposición, sino de añadir valor a la información. Vamos a ver a continuación sus funciones y tareas.

\subsection{Funciones}

Las funciones son las actividades propias de un oficio. Las del documentalista han evolucionado; veremos las tradicionales y las nuevas, que han surgido de la nueva sociedad en la cual vivimos, la sociedad del conocimiento.

\subsubsection{Funciones tradicionales}

Jacques Chaumier (1984) presenta de forma muy clara las funciones del documentalista:

- análisis de las necesidades de los usuarios/clientes: función que abre la cadena documental y ayuda a la reformulación (Chaumier, 1984; Michel, 1998);

— búsqueda de las fuentes, para conocerlas y ponerlas a disposición de los usuarios (Chaumier, 1984; Michel, 1998);

— adquisición: lo que permite constituir su fondo documental se adquiere por compra, intercambio o donación;

— selección: se trata de elegir según las necesidades de sus usuarios/clientes y de los objetivos del centro de documentación;

— registro: operación administrativa cuyo objetivo es hacer un inventario de los documentos entrados en el servicio;

— catalogación;

— análisis documental: se subdivide en condensación (resumen) e indización;

— almacenamiento: clasificación física de los documentos y memorización de los datos de las operaciones de análisis y catalogación;

— difusión orientada hacia los usuarios;

— control de la satisfacción de los usuarios/clientes y del funcionamiento interno.

\subsubsection{Nuevas funciones}

Jean Michel (1998) añade estas nuevas funciones:

- ser capaz de autentificar y validar las fuentes y los recursos: la cuestión de la pertinencia y de la confianza se ha vuelto primordial en el entorno de Internet;

- saber manipular los documentos electrónicos para ponerlos a disposición de los usuarios/clientes, cualquiera que sea su plataforma de trabajo;

Scire. $14: 1$ (en.-jun. 2008) 75-86. ISSN 1135-3716. 
- poder realizar nuevas operaciones de almacenamiento, archivo y capitalización activa de lo que es pertinente y útil con la idea de poder reexplotarlo;

- concebir, crear y realizar nuevos productos y servicios que pongan de manifiesto las potencialidades de Internet, que satisfagan a los usuarios/clientes y que valoren las competencias de los documentalistas;

- ayudar a los usuarios/clientes en las funcionalidades y recursos de Internet;

— organizar nuevas redes de acceso a la información, la documentación y el conocimiento.

Aquí vemos que el documentalista tiene dos nuevos tipos de funciones: por un lado, poseer habilidades en el tratamiento de los documentos electrónicos; por otro, como también comenta José Luis del Río Sadornil (2000), llevar a cabo el asesoramiento y guía de los usuarios.

Aparece la noción de metaconocimiento, en la cual participa el documentalista (Rodríguez, 2001), que debe tener la capacidad de crear sistemas para

- definir e implementar metodologías para la determinación del conocimiento necesario en el cumplimiento de los objetivos, desarrollando formas de difusión de los conocimientos y su reutilización;

- determinar la información y el conocimiento necesarios para el desarrollo tanto de los procesos como de los procedimientos de actividad;

- detectar e integrar la información externa con la producida en la propia organización.

\subsection{Implicación con la gestión del conocimiento}

Paralelamente a la evolución de la sociedad del conocimiento se desarrolló, según hemos analizado anteriormente, la gestión del conocimiento, y vemos que los documentalistas están vinculados a esta por sus nuevas funciones y, como se indica en la jornada De la gestión de la información a la gestión del conocimiento (ADBS, 2001), "las funciones de la gestión del conocimiento necesitan competencias y saberes propios de los profesionales de la información: análisis de las necesidades, búsqueda y tratamiento de la información, difusión y puesta a disposición, valoración...".

Asimismo se subrayan las oportunidades para el documentalista (ibídem, 2001):

- jugar un papel de experto funcional en la definición de los proyectos de gestión del conocimiento;

- contribuir a asegurar la perennidad de las aplicaciones de gestión del conocimiento;

— intervenir en la definición de los contenidos en función de los objetivos de utilización; 
— imponer nuevas prácticas e introducir nuevas profesiones (valor añadido por el profesional de la información en la gestión de proyectos, nuevos campos de actividad como el de la documentación técnica, publicaciones sobre sitios de intranet e Internet...).

Esto demuestra que la gestión del conocimiento se ha convertido en una herramienta para el documentalista. Además, sabiendo que comunicar ha pasado a ser la palabra clave de esta sociedad (Accart, 2000), es necesario organizar de forma adecuada la información para difundirla de modo que llegue a todos los niveles, y la gestión del conocimiento cumple este requisito.

Las nuevas funciones tienen que ver con añadir valor, crear productos y, dentro de la empresa, organizar el conocimiento de esta. Una estrategia para cumplir parte de estas funciones es la gestión del conocimiento. El hecho de que tenga un papel en el metaconocimiento demuestra la necesidad de que se utilice.

Además, Mackenzie incluye entre las actividades del documentalista para la gestión del conocimiento atender a la documentación interna, organizar flujos de conocimientos externos e integrar datos, documentos y conocimiento personal, enlazando políticas, recursos, actividades (García Jiménez, 2002). Un modelo de gestión del conocimiento se perfila a partir de patrones de conocimiento, objetos de conocimiento y perfiles de conocimiento, en todo lo cual la participación del profesional es imprescindible.

\subsection{Nuevas habilidades}

Las profesiones de la documentación integran continuamente nuevas técnicas y tecnologías de la información, y por consiguiente la competitividad está presente a nivel tanto interno como externo (Bouthillier, 2002), de modo que se hace imprescindible encontrar estrategias para seguir siendo competitivo.

Hemos visto que la gestión del conocimiento puede ser una estrategia, por un lado para realizar las nuevas funciones del documentalista y, por otro, para permitir a la empresa utilizar su conocimiento adecuadamente.

Para Drucker (ápud Silva y Cunha, 2002) los "trabajadores del conocimiento" son personas capaces de agregar conocimiento para incrementar la productividad y generar innovación. En la sociedad del conocimiento, los individuos son fundamentales (ibídem, 2002).

Bouthillier (2002) informa de que se buscan profesionales de la información con competencias tecnológicas y de gestión en nuevos dominios, como el multimedia o el de la gestión del conocimiento, lo que nos demuestra que gestión del conocimiento y profesional de la información son dos "conceptos" muy unidos, que la gestión es una estrategia para el documentalista.

Como afirma Pinto (2001), se debe estudiar al profesional desde el punto de vista humano, técnico y científico. Con respecto al primero, hay que destacar el es-

Scire. $14: 1$ (en.-jun. 2008) 75-86. ISSN 1135-3716. 
píritu altruista, las aptitudes en la comunicación interpersonal y el afán de actualización y formación permanente. En el plano técnico, el profesional debe tener sus propias estrategias de análisis y síntesis en el camino hacia la explotación y reorganización de la información esencial del original. Por tanto, los requisitos técnicos se centran en la pericia en la lectura, un profundo espíritu analítico, la capacidad de síntesis, el dominio de la terminología y la habilidad en las técnicas documentales. Desde el punto de vista científico, debe ser experto no solo en un determinado campo, sino también en el terreno documental, y el grado de adiestramiento en ambas áreas oscilará entre lo generalista y lo especializado.

\section{Conclusiones}

La sociedad del conocimiento refleja la importancia de los profesionales de la información a la hora de seleccionar, organizar y tratar la información que genera y maneja la organización.

La gestión del conocimiento es una herramienta que permite organizar la información y el conocimiento de una organización desde un ámbito evolutivo y dinámico. Hace posible controlar los flujos de información, centralizarlos, para aumentar la competitividad.

La gestión del conocimiento es una filosofía organizacional que no se puede implantar en un corto periodo de tiempo; al igual que ocurre con la política de calidad, se deben ver resultados que motiven su continuidad y se ha de intentar una perfecta armonización entre todos los que participan en ella.

Lo que Jacques Attali llamaba "el futuro del documentalista" se está haciendo realidad. La gestión del conocimiento se ha convertido en una metodología necesaria e imprescindible en el trabajo del documentalista para realizar algunas de sus funciones, como, por ejemplo, las nuevas operaciones de almacenamiento, archivo y capitalización activa de lo que es pertinente y útil con la idea de poder reexplotarlo, la búsqueda de información, la previsión y organización de métodos para captar el conocimiento... La gestión del conocimiento permite ser más competitivo dentro de la misma empresa y de cara al exterior. Por su parte, la gestión del conocimiento necesita las habilidades de los profesionales de la información para llevarse a cabo, y conduce al documentalista a tener nuevas habilidades y competencias: trabajo en equipo, conocimientos de otros idiomas, etcétera.

Para concluir, hay que decir que el importante papel de los infodocumentalistas en la estructuración y representación de la información les sitúa entre dos sistemas: el de generación y el de tratamiento automático de documentos. El gran reto es encontrar los mejores métodos y técnicas para extraer, traducir y representar el contenido documental de manera correcta, desambiguada y exhaustiva (Lancaster, 2001). 


\section{Notas}

(1) Puede verse un listado de iniciativas de e-learning en http://europa.eu.int/comm/ education/programmes/elearning/projects_en.html.

\section{Referencias}

Accart, Jean-Philippe (2000). Bibliothécaire, documentaliste: même métier? // BBF. 45:1 (2000) 88-93. http://www.enssib.fr/bbf/bbf-2000-1/11-accart.pdf (2004-03-10).

ADBS (2001). De la gestion de l'information à la gestion des connaissances. Journée d'Études. http://www.adbs.fr/uploads/journees/2555_fr.php (2004-06-24).

Al-Hawamdeh, S. (2002). Knowledge management: re-thinking information management and facing the challenge of managing tacit knowledge. Information Research, 8:1 (2002), paper 143. http://informationr.net/ir/8-1/paper143.html (2004-06-01).

Balmisse, G. (2003). Gestion des connaissances: outils et applications du knowledge management. París: Vuibert, 2002. [Compte-rendu de Jean-Philippe Accart. // BBF, 48:3 (2003)].

Bouthillier, France (2002). La concurrence dans les métiers de la documentation: réalité ou fiction? // BBF. 47:1 (2002) 36-41. http://www.enssib.fr/bbf/bbf-2002-1/05-bouthillier.pdf (2004-03-10).

Bustelo Ruesta, C.; Amarilla Iglesias, R. (2001). Gestión del conocimiento y gestión de la información. // Boletín del Instituto Andaluz de Patrimonio Histórico. Año 9, 34 (2001) 226-230.

Cañas, A. J., et álii (2000). Herramientas para construir y compartir modelos de conocimiento. http://www.coginst.uwf.edu/ acanas/Publications/HerramientasModelos/ A\%20Canas\%20Ponencia\%20WISE\%2099.htm (2004-07-06).

Caro, J. L., et álii (2002). Modelado workflow multi-hebra aplicado a la gestión de empresas turísticas. // IV Congreso Turismo y Tecnologías de la Información y las Comunicaciones (TuriTec 2002). http://www.turismo.uma.es/turitec/turitec2002/actas/ Microsoft\%20Word\%20-\%208.CARO.pdf (2004-06-01).

Chaumier, Jacques (1984). Travail et méthodes du / de la documentaliste. París: Entreprise Moderne, 1984.

Drucker, Peter (1996). La gestión en un tiempo de grandes cambios. Barcelona: Edhasa, 1996.

Esteban Navarro, M. Á.; Navarro Bonilla, D. (2003). Gestión del conocimiento y servicios de inteligencia: la dimensión estratégica de la información. // El profesional de la información. 12:4 (2003) 269-281.

García-Caso, I. (2002). La gestión del conocimiento: el nuevo paradigma para las empresas. [Madrid]: Robotiker, 2002. http://cdt.robotiker.es/cdt/articulos/mostrar_pdf.jsp?id=1 (2004-06-01).

García Jiménez, Antonio (2002). Organización y gestión del conocimiento en la comunicación. Gijón: Trea, 2002.

Lancaster, F. W.; Pinto, M. (coords.) (2001). Procesamiento de la información científica. Madrid: Arco/Libros, 2001.

Scire. $14: 1$ (en.-jun. 2008) 75-86. ISSN 1135-3716. 
Michel, Jean (1998). Les document@ listes et internet. // ABF Bulletin d'Informations. 182 (1998). http://www.abf.asso.fr/publications/bulletin/182/article8.html (2004-06-16).

Novak, Joseph Donald; Gowin, D. Bob (1988). Aprendiendo a aprender. Barcelona: Martínez Roca, D. L. 1988.

Pinto, M. (2001). El resumen documental: paradigmas, modelos y métodos. Madrid: Fundación Germán Sánchez Ruipérez, 2001.

Río Sadornil, José Luis del (2000). El documentalista en la sociedad de la información. // Primer Congreso Universitario de Ciencias de la Documentación. Teoría, historia y metodología de la documentación en España (1975-2000) (14-17 de noviembre de 2000). http://www.ucm.es/info/multidoc/multidoc/revista/num10/paginas/pdfs/Jlrio.pdf (2004-06-17).

Rodríguez Rovira, Josep M. a (2001). Una actuación profesional, un rol social. Gestores del metaconocimiento. // El Profesional de la Información. 10:12 (dic. 2001) 30-32.

Sánchez, M. ${ }^{a}$ J.; Doucet, A.-V. (2004). Gestión del conocimiento, eLearning, mapas conceptuales: mejora de la calidad de aprendizaje. // I Congreso Internacional sobre Tecnología Documental y del Conocimiento, Madrid, 2004.

Silva, Edna da; Cunha, Miriam Vieira da (2002). Los profesionales de la información y los dilemas educacionales del mundo globalizado. // Scire 8:2 (jul.-dic. 2002) 23-32. 